\title{
ENVIRONMENTAL EFFECTS IN THE \\ STELLAR POPULATIONS OF ELLIPTICAL GALAXIES
}

\section{J. R. LUCEY}

Department of Physics, University of Durham, Science Labs., South Road, Durham, DH1 3LE, UK. (John.Lucey@durham.ac.uk)

\section{Introduction}

While early work successfully modelled the stellar population of elliptical galaxies as old, single-age, metal-rich systems, there is evidence that a small amount of recent (i.e. a few Gyrs ago) star formation has occurred in some nearby ellipticals (O'Connell, 1980; Rose, 1985; Pickles, 1985; Bica, 1988). Elliptical galaxies reside in environments ranging from the dense cores of rich clusters to isolated field regions. It has long been suggested that the global properties of ellipticals depend on environment. For example, McClure and Van den Bergh (1968) noted apparent differences in the $380-450 \mathrm{~nm}$ colours between a set of cluster ellipticals and three from the field. Recent work has tended to support the view that the properties of ellipticals depend on environment (e.g. de Carvalho and Djorgovski, 1992). The most likely cause of these differences is the presence of a small amount of recent star formation in the field ellipticals. This environmental dependency may have important consequences for the use of ellipticals as distance indicators.

\section{Colour-Magnitude Studies}

U-V colours of ellipticals are well correlated with absolute magnitude (Baum, 1959; Sandage, 1972) and this colour-magnitude (CM) relation is considered to result from a mass-metallicity relation. Early work suggested that the form of the CM relation depended on the environment (Burstein, 1977). In the late 1970s Sandage and Visvanathan $(1978, \mathrm{SV})$ reported the results of an extensive study of the CM relation using $\mathrm{u}-\mathrm{V}$ colours. One of their 
goals was to explore the possibility of using this relation to map the nearby velocity field. In their analysis SV claimed that the mean $\mathrm{u}-\mathrm{V}$ colours, at a given absolute luminosity, of field and cluster early-type galaxies were similar and hence argued that the CM relation could be used reliably to measure relative distances. In an influential paper, Larson, Tinsley and Caldwell (1980) reanalysed the SV dataset and concluded that there were systematic differences between the field and cluster ellipticals (see their Fig. 2). Whereas cluster ellipticals define a tight, well-defined CM relation, field ellipticals have a much weaker relation and at a fixed absolute magnitude are systematically bluer in $\mathrm{u}-\mathrm{V}$ by 0.06 magnitude. They concluded that the bluer colours of field ellipticals were probably due to the presence of a small amount of recent star formation. Aaronson, Persson and Frogel (1981) also found differences in the V-K colours of early-type galaxies in the Coma and Virgo clusters. They concluded that the CM relation was non-universal. These two later studies cast serious doubt on the use of the $\mathrm{CM}$ relation as a distance indicator and led to the emphasis being placed on other relationships, e.g. luminosity versus central velocity dispersion $(\sigma)$. A recent study with improved colour data for Virgo and Coma early-type galaxies, in contrast to Aaronson, Persson and Frogel, finds that the CM relations for these two important clusters are in good agreement (Bower et $a l ., 1992 \mathrm{a}, \mathrm{b})$. The Coma ellipticals in this study lie in the central region of the cluster. They show a remarkably small scatter about the CM relation which implies a high degree of uniformity in their star formation histories.

\section{Line Strength Studies}

While the $M g_{2}$ index has been used by many authors as a metallicity indicator it is also sensitive to the mean age of the stellar population. Whereas both Terlevich et al. (1981) and Tonry and Davis (1981) found only a very weak correlation between luminosity and $\mathrm{Mg}_{2}$, Dressler (1984) found that ellipticals in the Virgo and Coma clusters displayed a strong relation. The luminosity versus line strength relation was investigated further by Bica and Alloin (1987) who used the average equivalent width of the $M g+M g H$ and $C N$ absorption features as a metallicity index. They found that early-type galaxies in the field had weaker line strengths compared to similar luminosity galaxies in the Virgo and Fornax clusters. They concluded that field early-type galaxies possessed an intermediate age stellar population that was not present in cluster galaxies. The line strength studies thus show a similar trend to that seen in the colour data.

The photometric and spectroscopic survey of over 400 ellipticals by the 7-Samurai (Faber et al., 1989) allowed new tests to be made. To assess the degree of correlation between the mass parameters and the stellar popula- 
tion properties, Burstein et al. (1988) examined the relationship between the $M g_{2}$ index and $\sigma$. They concluded that while most ellipticals followed a well-defined $\mathrm{Mg}_{2}-\sigma$ relationship, a significant number of ellipticals $(\sim 15 \%)$ were found that had weaker values of $M g_{2}$ at a given $\sigma$ (see their Fig. 1). These $M g_{2}$-weak ellipticals were attributed to either the presence of low amounts of recent star formation or real chemical composition differences.

For a sample of ellipticals, mostly located in the field and small groups, Schweizer et al. (1990) found that, at a given absolute magnitude, the colours and $M g_{2}, H \beta$ and $C N$ line strengths were correlated with the amount of fine structure (i.e. the presence of ripples). They showed that these correlations were probably the result of systematic differences in mean stellar age, in the sense that the ellipticals that have the most fine structure show the most evidence for younger stellar ages. These younger ellipticals have, on average, $M g_{2}$ values that are about $0.02 \mathrm{mag}$ lower at a given $\sigma$ compared with other ellipticals (Bender, Burstein and Faber, 1993). Hence the position of an elliptical in an $M g_{2}-\sigma$ plot may be used as a rough indicator of mean stellar age.

We (Guzman et al., 1992) compared the $M g_{2}-\sigma$ relationships of ellipticals that reside in different parts of the Coma cluster. Unlike previous work, our study included ellipticals that lie out at the turnaround radius of the cluster (i.e. $\sim 5^{\circ}$ from the cluster centre) as well as ellipticals from the dense cluster core. Thus we surveyed a large range of galaxian density. The ellipticals in the cluster core region showed a well-defined $M g_{2}-\sigma$ relationship whereas those from the outer parts of the cluster were significantly offset to lower $\mathrm{Mg}_{2}$ at a given $\sigma$. Between the core and far halo samples we measured an offset of $0.017 \pm 0.005$ mag (see Fig. 1).

We showed that this offset was consistent with the outer ellipticals having a small amount of recent star formation. In Fig. 2, the rich cluster and field $M g_{2}-\sigma$ relations are shown for Burstein's Mark II dataset.

The field sample is offset to lower $M g_{2}$ values by $0.014 \pm 0.003 \mathrm{mag}$. However, the systematic uncertainties in matching the different observing systems of the Mark II dataset and Malmquist bias effects make the significance of this offset difficult to assess reliably. Overall, the $M g_{2}-\sigma$ relation does support the existence of a small environmental effect that is consistent with the presence of a small amount of recent star formation occurring in ellipticals that reside in low density environments.

By using a set of spectral indicators in the blue, Rose and collaborators have developed a set of tools to measure the mean age of stellar populations of ellipticals (Rose et al., 1995). Their spectral indicators measure the mean surface gravity of the integrated light at $4000 \AA$ and were designed to enable the effects of metallicity and age to be disentangled. Bower et al. (1990) compared the line strengths of early-type galaxies in high- and 

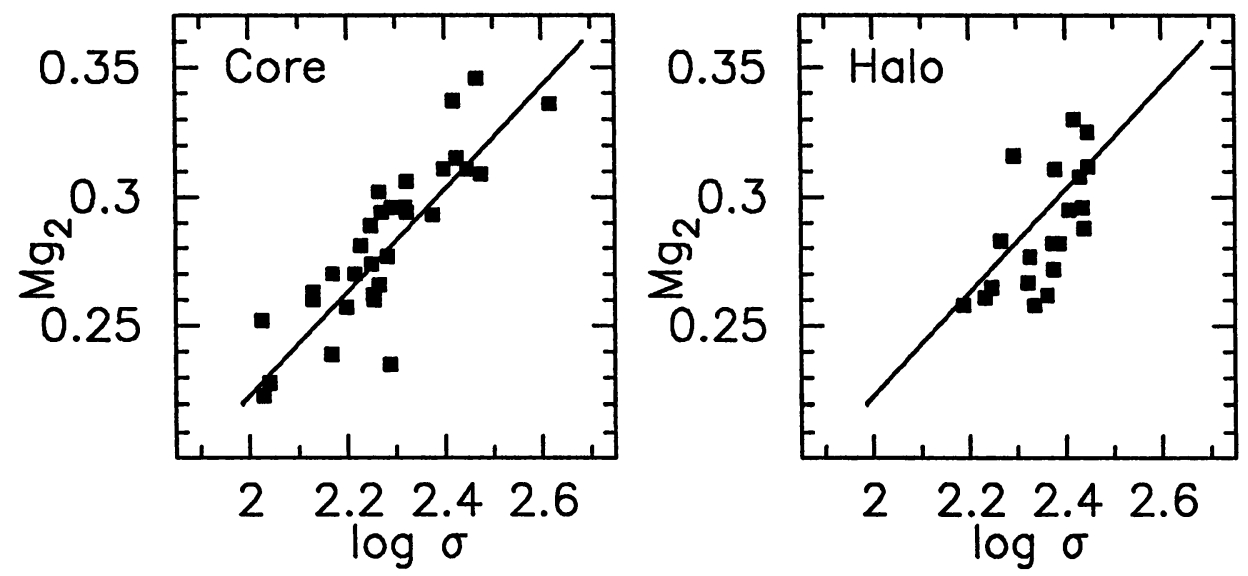

Figure 1. Comparison of $M g_{2}-\sigma$ relation for core and halo ellipticals in the Coma cluster (from Guzman et al.)
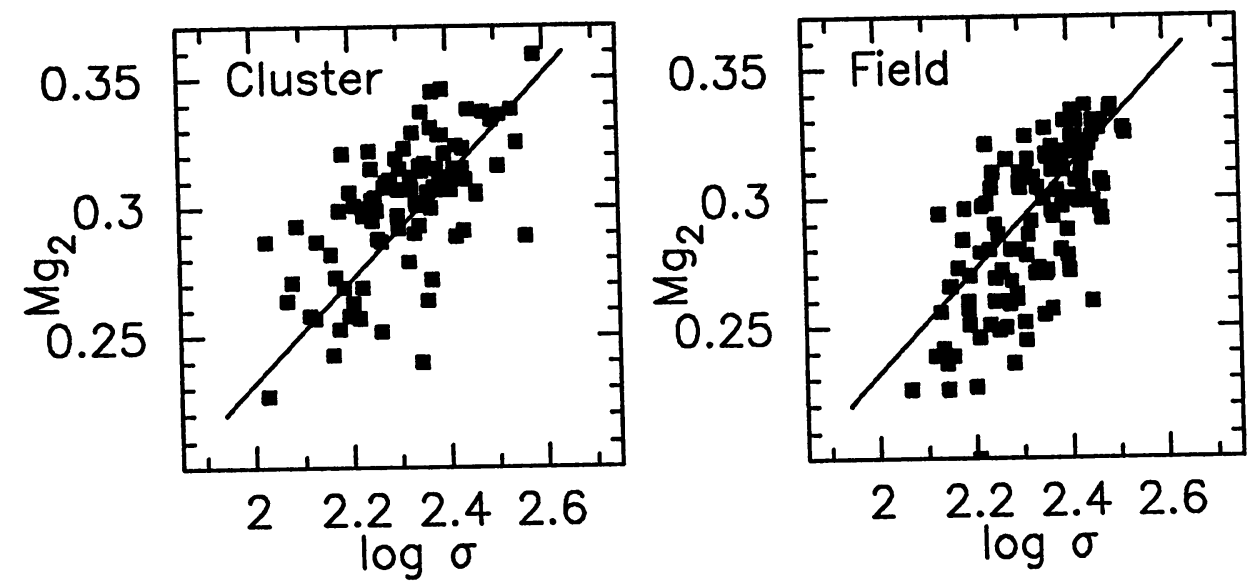

Figure 2. Comparison of the $M g_{2}-\sigma$ relation for the rich cluster and field ellipticals from Burstein's Mark II dataset. The lack of ellipticals with $\log \sigma<2.1$ in the field sample is a selection effect

low-density environments. They found evidence that star formation continued to more recent epochs in field early-type galaxies as compared to those located in rich clusters. Caldwell et al. (1993) used multi-fibre spectroscopy to investigate the star formation properties of early-type galaxies in two regions of Coma; a cluster core field and a field 40 arcmin SW of the cluster centre. Their outer field included the NGC 4839 subcomponent. Nearly 
all galaxies in the central field were found to have the spectra typical of an old stellar population, with little signs of either recent star formation or nuclear activity. In their outer field about one-third of the sample showed spectral features that indicated recent star formation or nuclear activity. This may be the detection of a real environmental trend or may reflect activity induced by the interaction of the subcomponent with the main body of the cluster.

\section{Environmental Biases on the $D_{n}-\sigma$ Distance Indicator}

The $D_{n}-\sigma$ relationship has been used to map the local peculiar velocity field (Faber et al., 1988). The effect of stellar population differences on the distances derived with $D_{n}-\sigma$ was investigated by Burstein, Faber and Dressler (1990). They examined the dependence of $\log _{10}(V / D)$ on cluster richness, where $V$ is the measured redshift and $R$ is the derived distance. If the environmental differences did cause a bias then the distances for field ellipticals would be, on average, offset from rich cluster ellipticals. They found no offset (see their Fig. 8) and hence concluded that the derived distances were not significantly affected by the environment. While Burstein, Faber and Dressler do not explicitly give an uncertainty on this conclusion, using the same Mark II dataset I derived a $\log _{10}(V / D)$ offset of $0.02 \pm 0.02$ dex between the richest cluster ellipticals and those in the field. To directly assess the environmental dependence of the $D_{n}-\sigma$ relation, we (Lucey et al., 1991) compared the properties of a sample of ellipticals from the densest parts of Coma with those that lie out near the cluster turnaround radius. We measured a $D_{n}-\sigma$ zero-point difference of only $0.02 \pm 0.04$ dex. Hence the effect of the environment on the $D_{n}-\sigma$ distance indicator appears to be small.

The ellipticals in Schweizer et al.'s sample that exhibit the most fine structure also have the clearest evidence for the presence of a younger stellar population. As derived from the $D_{n}-\sigma$ relation, these ellipticals have systematically positive peculiar velocities (Gregg, 1992; Schweizer and Seitzer, 1992). The ellipticals with most fine structure (i.e. $\Sigma>4$ ) have an average $\log _{10}(V / D)$ of $\sim 0.15$ dex. These ellipticals are extreme cases and hence this value would seem to be an upper limit to a bias caused by stellar population differences. More distorted and younger galaxies would probably not be classified as ellipticals. De Carvalho and Djorgovski (1992) have emphasised that field ellipticals do not follow the same distance indicator relations as rich cluster ellipticals. While they do not explicitly state how much the $D_{n}-\sigma$ (or Fundamental Plane, FP) indicator is likely to be affected this can be estimated from their Fig. 1. The offset in $\log r_{e}$ between the FP of their cluster and field samples is $\sim 0.2 \mathrm{dex}$. This value seems to 
be inconsistent with the offsets estimated by both Burstein, Faber and Dressler, and Lucey et al..

The agreement between the peculiar velocity maps derived independently from spiral and elliptical galaxy samples suggests that any environmental effect on $D_{n}-\sigma$ is relatively small (Kolatt and Dekel, 1994). However Guzman (1993a,b) has noted that the $M g_{2}-\sigma$ relation for the early-type galaxies in the Hydra-Centaurus (HC) region is significantly offset, i.e. by $0.017 \pm 0.004 \mathrm{mag}$ in $M g_{2}$, relative to samples from rich northern clusters. He suggests that if this offset results from recent star formation, then some fraction of the large positive peculiar velocities observed in $\mathrm{HC}$ is spurious (c.f. Kaiser, 1988; Silk, 1989). While it is plausible that some of the observed offset may be caused by systematic errors in the $M g_{2}$ and $\sigma$ measurements this seems unable to account for all of this effect.

\section{Conclusions}

Ellipticals that reside in the densest regions of rich clusters are relatively homogeneous. Their colours and line strengths suggest that their star formation terminated at a relatively early epoch. In contrast ellipticals that reside in low density regions have a heterogeneous character, which is consistent with some of these galaxies possessing a small amount of recent star formation. There is disagreement on the amount by which this observed difference biases FP-based distance indicators, such as $D_{n}-\sigma$. Regardless of the size of this effect, correction procedures are possible. We (Guzman and Lucey, 1993) have described a simple scheme that uses the observed residuals from the $M g_{2}-\sigma$ relation to estimate the likely bias in the $D_{n}$ diameter. Clearly other more sophisticated schemes using more reliable star formation indicators like $H \beta$ or Rose indicators could be developed. Alternatively the development of a K-band FP distance indicator should offer significant advantages for reducing the biases caused by the stellar population differences between cluster and field ellipticals.

\section{References}

Aarsonson, M., Persson, S.E. and Frogel, J.A., 1981, Ap. J. 245, 18

Baum, W.A., 1959, P. A. S. P. 71, 106

Bender, R., Burstein, D. and Faber, S.M., 1993, Ap. J. 411, 153

Bica, E. and Allion D., 1987, A. \& $A .181,270$

Bica, E., 1988, A. \& A. 195, 76

Bower, R.G., Ellis, R.S., Rose, J.A. and Sharples, R.M., 1990, A. J. 99, 530

Bower, R.G., Lucey, J.R. and Ellis, R.S., 1992a, M. N. R. A. S. 254, 589

Bower, R.G., Lucey, J.R. and Ellis, R.S., 1992b, M. N. R. A. S. 254, 601

Burstein, D., 1977, Evolution of Galaxies and Stellar Populations, Eds. Tinsley, B.M. and Larson, R.B., Yale University Observatory, New Haven, p. 191

Burstein, D., Davies, R.L., Dressler, A., Faber, S.M., Lynden-Bell, D., Terlevich, R.J. 
and Wegner, G., 1988, Towards Understanding Galaxies at Large Redshift, Eds. Kron, R.G. and Renzini, A., Kluwer Academic Publishers, Dordrecht, p. 17

Burstein, D., Faber, S.M. and Dressler, A., 1990, Ap. J. 354, 18

Caldwell, N., Rose, J.A., Sharples, R.M., Ellis, R.S, and Bower, R.G., 1993, A. J. 106, 473

de Carvalho, R.R. and Djorgovski, S., 1992, Ap. J. 389, L49

Dressler, A., 1984, A. J. 281, 512

Faber, S.M., Wegner, G., Burstein, D., Davies, R.L., Dressler, A., Lynden-Bell, D. and Terlevich, R.J., 1989, Ap. J. Suppl. 69, 763

Gregg, M.D., 1992, Ap. J. 384, 43

Guzman, R., 1993a, Cosmic Velocity Fields, Eds. Bouchet, F.R. and Lachieze-Rey, M., Editions Frontieres, France, p. 545

Guzman, R., 1993b, PhD thesis, University of Durham

Guzman, R., Lucey, J.R., Carter, D. and Terlevich, R.J., 1992, M. N. R. A. S. 257, 187

Guzman, R. and Lucey, J.R., 1993, M. N. R. A. S. 263, L47

Kaiser, N., 1988, Large-Scale Structure and Motions in the Universe, ICTP

Kolatt, T. and Dekel, A., 1994, Ap. J. 428, 35

Larson, R.B, Tinsley, B.M. and Caldwell, C.N., 1980, Ap. J. 237, 692

Lucey, J.R., Guzman, R., Carter, D. and Terlevich, R.J., 1991, M. N. R. A. S. 253, 584

McClure, R.D. and Van den Bergh, S., 1968, A. J. 73, 313

O'Connell, R.W., 1980, Ap. J. 236, 430

Pickles, A.J., 1985, Ap. J. 296, 340

Rose, J.A., 1985, A. J. 90, 1927

Rose, J.A., Bower, R.G., Caldwell, N., Ellis, R.S., Sharples, R.M. and Teague, P., 1995, preprint

Sandage, A., 1972, Ap. J. 176, 21

Sandage, A. and Visvanathan, N., 1978, Ap. J. 223, 70

Schweizer, F., Seitzer, P., Faber, S.M., Burstein, D., Dalle Ore, C.M. and Gonzalez, J.J., 1990, Ap. J. 364, L33

Schweizer, F. and Seitzer, P., 1992, A. J. 104, 1039

Silk, J., 1989, Ap. J. 345, L1

Terlevich, R.J., Davies, R.L, Faber, S.M. and Burstein, D., 1981, M. N. R. A. S. 196, 381

Tonry, J.L. and Davis, M., 1981, Ap. J. 246, 680

WHITE: It is a generic feature of hierarchical clustering models that ellipticals in the field should be somewhat younger than ellipticals in dense environments. My impression is that all the possible trends which you have highlighted suggest differences in age in this sense. Is this correct?

LUCEY: Yes. All the observed trends are consistent with field ellipticals having, on average, younger stellar ages. 